\title{
Fast Multi-scale imaging using the Beamline I13L at the Diamond Light Source
}

C. Rau, M. Storm, S. Marathe, A. Bodey, M. Zdora, et al.

C. Rau, M. Storm, S. Marathe, A. J. Bodey, M. Zdora, S. Cipiccia, D. Batey, X. Shi, S. M. L. Schroeder, G. Das, M. Loveridge, R. Ziesche, B. Connolly, "Fast Multi-scale imaging using the Beamline I13L at the Diamond Light Source," Proc. SPIE 11113, Developments in X-Ray Tomography XII, 111130P (1 October 2019); doi: 10.1117/12.2543405

SPIE Event: SPIE Optical Engineering + Applications, 2019, San Diego, California, United States 


\title{
Fast Multi-Scale Imaging using the Beamline I13L at the Diamond Light Source
}

\author{
C. Rau* ${ }^{* 1,2,3}$, M. Storm ${ }^{1}$, S. Marathe ${ }^{1}$, A.J. Bodey ${ }^{1}$, M. Zdora ${ }^{1,4,5}$, S. Cipiccia ${ }^{1}$, D. Batey ${ }^{1}$, X. Shi ${ }^{1}$, \\ S.L.M. Schroeder ${ }^{1,6,7}$, G. Das ${ }^{1,6,7}$, M. Loveridge ${ }^{8}$, R. Ziesche ${ }^{9}$, B. Connolly ${ }^{10}$. \\ ${ }^{1}$ Diamond Light Source Ltd, Harwell Science and Innovation Campus, Didcot, OX 11 ODE, UK \\ ${ }^{2}$ University of Manchester, School of Materials Grosvenor St., Manchester, M1 7HS, UK \\ ${ }^{3}$ Northwestern University, 303 E. Chicago Avenue, Chicago, IL 60611-3008, USA \\ ${ }^{4}$ Department of Physics \& Astronomy, University College London, London WC1E 6BT, UK \\ ${ }^{5}$ Department of Physics \& Astronomy, University of Southampton, Southampton, SO17 1BJ, UK \\ ${ }^{6}$ School of Chemical and Process Engineering, University of Leeds, Leeds, LS2 9JT \\ ${ }^{7}$ Continuous Manufacturing and Crystallisation Group, Research Complex at Harwell, \\ Harwell Science and Innovation Campus, Didcot, OX 11 0DE, UK \\ ${ }^{8}$ WMG, University of Warwick, Gibbett Hill Road, Coventry CV4 7AL, UK \\ ${ }^{9}$ Department of Chemical Engineering, University College London, London, WC1E 7JE, UK \\ ${ }^{10}$ Corrosion \& Protection Centre, Department of Materials, University of Manchester, \\ Manchester M13 9PL, UK
}

\begin{abstract}
The DIAMOND beamline I13L is dedicated to multi-scale and multi-modal imaging in real and reciprocal space. The beamline consists of two independently operating experimental stations, located at a distance of more than $200 \mathrm{~m}$ from the source. The Imaging Branch performs micro-tomography with in-line phase contrast in the 6-30 keV energy range. In addition, a grating interferometry setup and a full-field microscope for nano-tomography are currently implemented. Other techniques providing high-resolution three-dimensional information, in particular coherent X-ray diffraction, are hosted on the Coherence Branch. All imaging methods are tested to operate with large energy bandwidths and therefore shorter exposure times. To this end, two options are currently used: the so-called 'pink-beam' mode using a reflecting mirror and X-ray filters and monochromatic mode using a multilayer monochromator. The operation mode enables science for in-situ and operando studies across a wide range of scientific areas.
\end{abstract}

Keywords: tomography, synchrotron radiation, nano-imaging, phase contrast, X-ray microscopy, ptychography, Bragg-CDI, pink-beam

\section{INTRODUCTION}

Imaging with hard X-rays in the energy range between 6-30 keV, is routinely carried out on millimeter sized objects with micrometer resolution at the I13L beamline. The high photon flux available at synchrotron radiation sources allow for in-situ and operando studies to be carried out with dedicated sample environments. Imaging at higher spatial resolution is achieved with a variety of microscopy methods, working either in direct or reciprocal space.

While micro-tomography is well established on the I13L beamline, high-resolution experiments have seen more recent developments and are currently being explored extensively for scientific applications. Grating interferometry provides phase and small angle information with micrometer resolution, the full-field microscope provides sub-100 nm resolution over a field of view up to $100 \mu \mathrm{m}$ and ultimate resolution and sensitivities are achieved with ptychography and Bragg CDI.

Short recording times are essential for the usability of these imaging methods, especially for operando measurements. By increasing the energy bandwidth of the radiation from 'monochromatic' (in this case with a $\mathrm{Si}(111)$ crystal

* christoph.rau@diamond.ac.uk, phone +44 1235778796

Developments in X-Ray Tomography XII, edited by Bert Müller, Ge Wang, Proc. of SPIE Vol. 11113, 111130P - (C) The Authors. Published under a Creative Commons Attribution CC-BY 3.0 License · doi: 10.1117/12.2543405 
monochromator) with $\Delta E / E=10^{-4}$ to the energy bandwidth of an undulator harmonic typically increases the flux by about two orders of magnitude. The robustness of each imaging technique in regards to the increased energy bandwidth has been tested experimentally. For all methods we are aiming at achieving tomography scanning times in minutes or seconds.

The beamline addresses a large user community for multi-scale and multi-modal science. User friendliness and ease for data analyses are implemented by the SAVU software pipeline [1] and in particular for ptychography by the PtyREX software [2]. Users may visit after experiments to conduct data analyses (visualisations, segmentations, measurements, etc.) at the I13 Data Beamline [3].

\section{THE BEAMLINE}

The I13L beamline is located in one of Diamond's long straight sections of the $3 \mathrm{GeV}$ storage ring. The so-called 'mini-beta' layout permits the independent operation of the two branchlines with small gaps of the undulator sources. Each branchline is equipped with a series of slits, X-ray filters, horizontal deflecting mirrors and Si (111) monochromators. The Diamond-Manchester Imaging Branchline also operates with a multilayer monochromator, with different sets of multilayer stripes. On the Coherence Branchline the lateral coherence length can be adapted with front-end slits and a CRL focusing optic located in the first optics hutch. More details can be found elsewhere [4, 5].

\subsection{Pink beam imaging}

The beamline is designed for experiments requiring large lateral coherence lengths. Regarding the longitudinal coherence length (in other words the monochromaticity of the radiation) the requirements may be more relaxed for certain experimental methods. We carried out tests to examine the influence of energy bandwidth on image quality. We have explored two different broadband modalities. 'Pink-beam' includes few or one undulator harmonics by combining a low-pass mirror and a high-pass filter. Alternatively, moderate energy bandwidth is achieved with a multilayer monochromator. Both are briefly described in the following and the features of each are discussed.

The radiation generated by an undulator source comprises a series of intensity peaks as a function of X-ray photon energy, called undulator harmonics (see figure 1, yellow line). Each harmonic typically has a bandwidth of a few percent, and the separation of the harmonics increases as energy of the electron storage ring is increased. At highenergy storage rings single harmonics can be filtered out by a band-pass using X-ray filters and a reflecting X-ray mirror [6]. The filter cancels lower photon energies, filter materials require a reasonable thermal conductivity to withstand the heat load. Typical materials used are carbon and aluminum for broadband filtering, or silver,

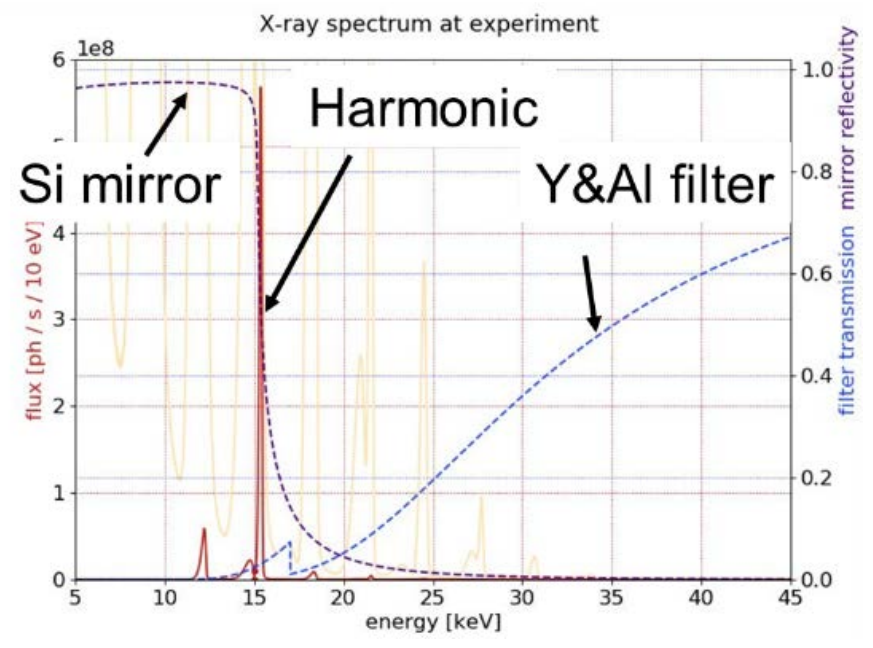

Figure 1: Simulated pink-beam spectrum for Diamond-Manchester Imaging Branchline (simulation M. Storm).

Undulator spectrum in yellow, transmitted harmonic in red, mirror reflectivity in purple, filter transmission in blue.

molybdenum and palladium for selective filtering around their absorption edges. Some filters help to single out a particular harmonic with an absorption edge in the vicinity of the harmonic. Higher photon energies are rejected by a 
reflective mirror: for X-rays the reflective angles are shallow and the cut-off energy can be modulated by the incident angle of the X-rays onto the mirror or by the mirror coating material (typically ruthenium or platinum). Overall the method is relatively simple and efficient. The filter structure and impurity/degradation traces on the mirror combined with position instabilities are the main contributing factor to the degradation of the beam quality/coherence. Additional structures in the beam profile can be corrected while the profile itself is stable over time.

\subsection{Multilayer monochromator}

Multilayer monochromators (MLMs) are used for other synchrotron radiation sources including wigglers and bending magnets. MLMs typically provide an energy bandwidth $\Delta E / E$ of about $10^{-2}$, which corresponds approximately to the energy bandwidth of an undulator harmonic. The multilayer consists of a system of alternating high and low refractive index materials. In general a striped structure is added to the beamprofile [7, 8]: difficult to correct when the monochromator is not stabl. The central bandpass energy can be easily selected by tuning the Bragg angle.

\section{EXPERIMENTS}

\subsection{Diamond-Manchester Imaging Branchline}

The Diamond-Manchester Imaging Branchline contains three experimental setups for imaging in direct space: micro-tomography with in-line phase contrast, grating interferometry and a full-field microscope.

\subsubsection{Micro-tomography setup}

The micro-tomography setup on the Imaging Branch is operated mostly in pink-beam mode, typically with an undulator gap down to $5 \mathrm{~mm}$ and a combination of $1.3 \mathrm{~mm}$ glassy carbon and 2-3 mm aluminum filters. When operating at higher photon energies (above $25 \mathrm{keV}$ ) about $100 \mu \mathrm{m}$ steel is added to the filter stack. Structures with weak absorption contrast are enhanced by increasing the distance between sample and detector. Performing phase retrieval with the Paganin filter for example, assumes an average photon energy. A typical example for an operando experiment recorded with pink beam is shown in figure 2. The study concerns multiphase flow reactive transport processes during injection of supercritical $\mathrm{CO}_{2}$, with the aim of obtaining a better understanding of the storage of $\mathrm{CO}_{2}$ in sandstone in the presence of brine [9-13]. The study revealed new mechanisms, namely a snap-off of brine connectivity under high-loading conditions.

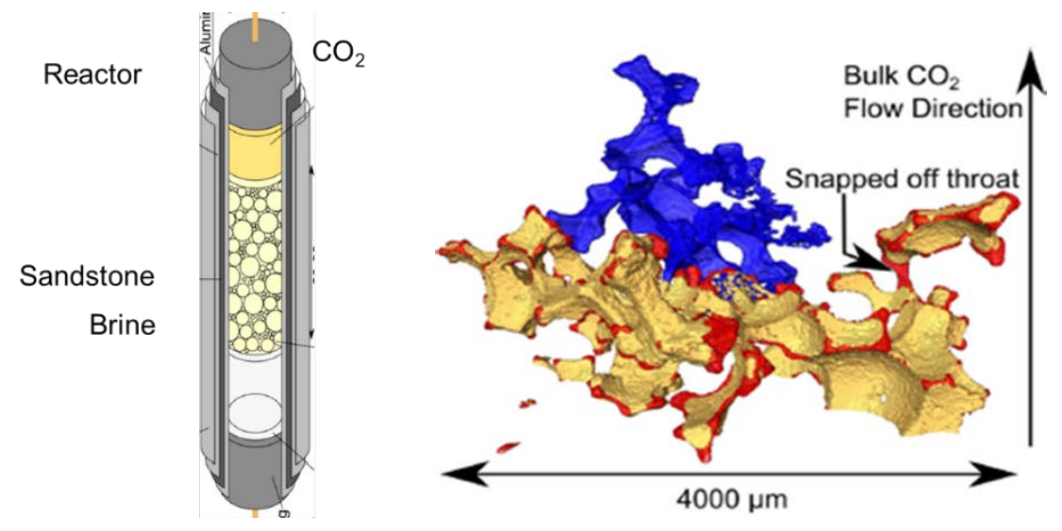

Figure 2: Imaging of dissolving $\mathrm{CO}_{2}$ in a sandstone-brine matrix with pink-beam.

Left: Reactor to mix components. Right: Drainage of the brine by $\mathrm{CO}_{2}$.

The $\mathrm{CO}_{2}$ (yellow) expands to new areas (blue) and recesses partially (red). Some $\mathrm{CO}_{2}$ volumes are snapped-off [9-13].

\subsubsection{Grating interferometer}

Grating interferometry resolves structures spatially, similar to in-line phase contrast imaging, on the micrometer scale $[14,15]$. The method provides additional information channels such as phase shift and small angle scattering. The phase signal provides higher sensitivity than absorption and data can be segmented with more ease [16-19].

The small angle scattering (dark-field) signal is explored for distinguishing materials of similar refractive index but different structure on the nano and Angstrom lengthscales. While in-line phase contrast identifies defined objects, 
small angle scattering in general is sensitive to electronic fluctuations before the formation of a separate phase happens. This is in particular interesting when observing phase separations or the crystallization in materials and processes proceeding a phase transition. In our most recent work we studied the formation of crystals using pink beam.

The requirements regarding the energy bandwidth for grating interferometry are moderate and the influence has been tested [20] using the energy spectrum as shown in figure 1 . The resulting exposure times are about $5 \mathrm{~ms}$ and, with a single grating geometry, time-resolved data can be recorded without any additional scanning.

Figure 3 shows a first result from a study of anti-solvent crystallization studies of lovastatin (in water acetone solution) with anti-solvent of water. The mixing zone is in the centre of the image, details about the geometry of the experimental setup can be found elsewhere [20]. The image series of the dark-field and also of the phase shift channel reveal the moment preceding the crystallization in a specific zone. The experiment was carried out at an average photon energy of about $19 \mathrm{keV}$, the exposure time per image was $5 \mathrm{~ms}$ and the whole process was recorded over $7 \mathrm{sec}$. The results are important for the pharmaceutical industry in understanding the formation of basic medication components.

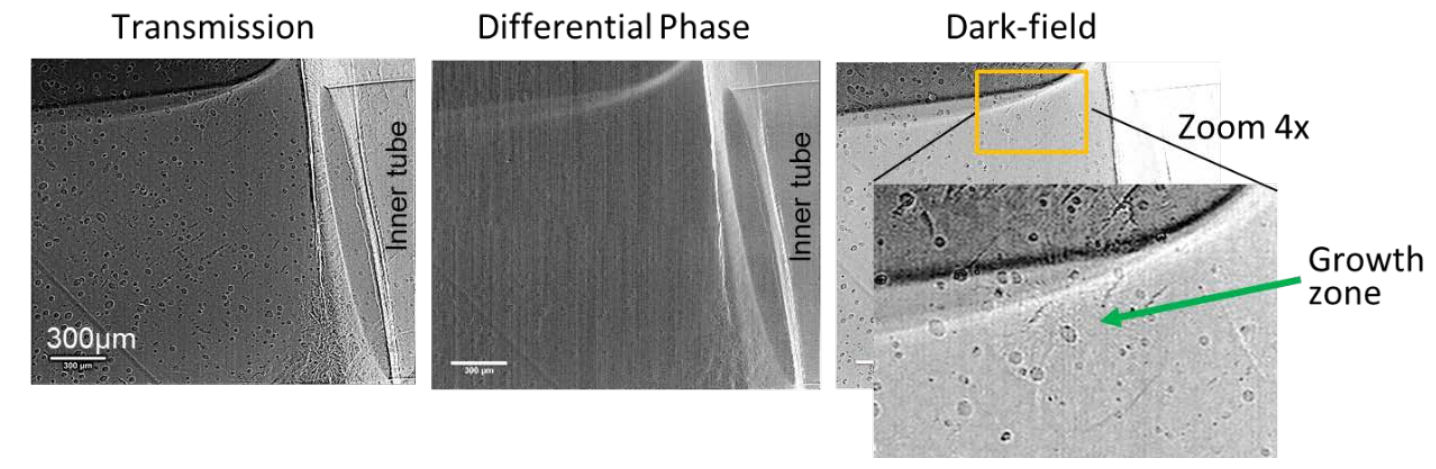

Figure 3: Single exposure during the crystallization process of a water-acetone mix. In the dark-field image (right side) the formation of crystals can be anticipated, the crystals will be deposited in a thin film growth.

\subsubsection{Transmission X-ray microscope}

The transmission X-ray microscope (TXM) at the I13 beamline is designed to explore spatial resolutions below $100 \mathrm{~nm}$ in direct space [21-23]. The X-ray microscope consists of similar elements as a visible light microscope. The condenser optic illuminates the sample and matches the numerical aperture of the objective for optimal contrast. The objective lens projects the magnified image of the sample onto the detector. Optical schemes known from visible light microscopy, such as the Zernike phase contrast, are adaptable to X-rays. For the I13 microscope a 'beam-shaper' is used as condenser optic [24] to achieve a field of view to about 50-100 $\mu \mathrm{m}$ side length. The beam-shaper pattern is specifically designed for a modified illumination scheme in Zernike phase contrast. The objective lenses are optimized for the energy range between $8-13 \mathrm{keV}$ and their resolution limit is $60 \mathrm{~nm}$.

The optimal energy bandwidth for the microscope is inversely proportional to the number of zones $(\mathrm{N})$ in the objective zone plate: $\Delta E / E \sim 1 / N$. A zone plate with 500 zones should therefore have an energy bandwidth of $2 \times 10^{-3}$. Note that the energy bandwidth of a monochromator with $\mathrm{Si}(111)$ crystals is typically $10^{-4}$. We examined experimentally the influence of energy bandwidth on the image quality, using different stripes of the multilayer monochromator. In figure 4, a TXM comparison between a $1 \%\left(\mathrm{Mo} / \mathrm{B}_{4} \mathrm{C}\right.$ system) and $5 \%\left(\mathrm{Ru} / \mathrm{B}_{4} \mathrm{C}\right.$ system) energy bandwidth is shown. For the latter the full undulator harmonic intensity is transmitted (which is typically 2-3\%). For resolving $100 \mathrm{~nm}$ structures the energy bandwidth needs to be restricted to below $1 \%$. The exposure times for the microscope have been reduced from minutes per projection to below one second, mainly due to the increased flux by the multilayer monochromator and the use of a Hamamatsu C12849-101U, a sCMOS camera with a $10 \mu \mathrm{m}$ Gadox scintillation screen. The flux on the microscope can be further increased by some adaptations of the beamline optics. Tomographic scans can now be carried out within some minutes. In-situ and operando investigations can be carried out. The deterioration of photonic crystals under thermal treatment has been studied. More recently a dedicated sample environment has been developed to study the corrosion of a carbon steel in an aggressive environment, which is important for the petrol industry. 


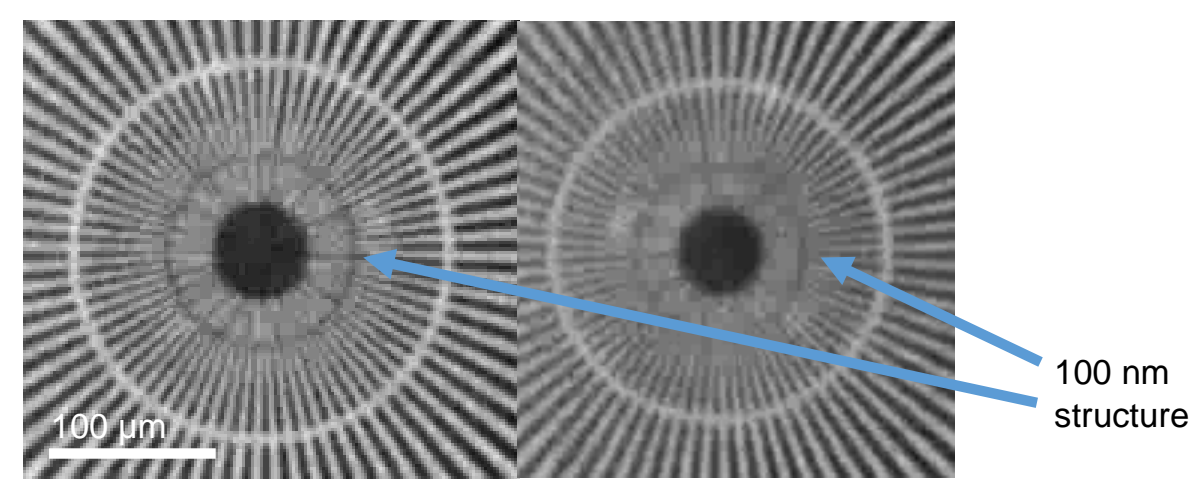

Figure 4: Comparison of TXM operation using different bandpass of the monochromator's multilayer systems. Left: Mo-B4C strip with $1 \%$ bandwidth, right Ru-B4C with 5\% bandwidth. $100 \mathrm{~nm}$ structures are visible on the left image.

A $40 \mu \mathrm{m}$ thick X65 steel rod was exposed over two days to a $\mathrm{CO}_{2}$ saturated emulsion at $80^{\circ} \mathrm{C}$ with various flow rates, observing the development of the corroded layer (see figure 5).
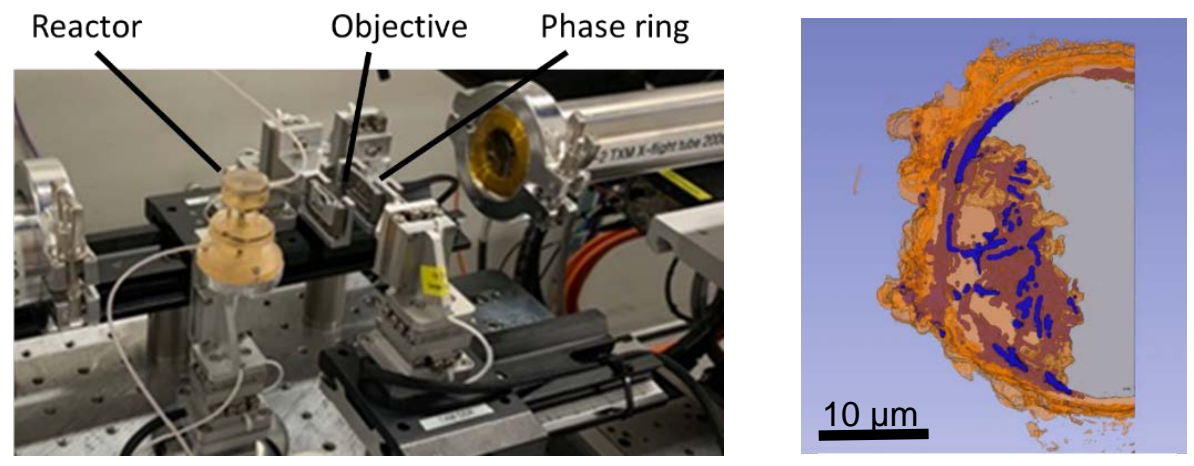

Figure 5: Left: TXM with dedicated sample environment, here an in-situ flow cell, to study corrosion as a function of temperature, solution chemistry, and exposure time. Right: Deterioration of a $40 \mu \mathrm{m}$-thick X65 steel pin in a $\mathrm{CO}_{2}$ saturated brine solution, heated at $80^{\circ} \mathrm{C}$. The formation of a oxide scale and a localized corrosion site can be observed. All images courtesy Brian Connolly, Manchester University.

\subsection{The Coherence Branchline}

The Coherence Branchline hosts ptychography and Bragg-CDI as the main user experiments and offers the possibility of carrying out bespoke instrumentation experiments requiring large coherence lengths. 3-D information is recoded at the nanoscale using ptychography in a similar fashion to micro-tomography, but where each projection is reconstructed from a 2D scan. As well as high resolution structural information in the transmission mode, the Bragg modalities also reveal highly sensitive strain information, where the sample is scanned around the Bragg peak.

\subsubsection{Ptychography and Bragg-CDI}

Recording ptycho-tomographic data takes currently about 10 hours, scanning the sample across a pencil beam with a rate of some tens $\mathrm{Hz}$, covering an area of about $3 \mu^{2} \mathrm{~s}^{-1}$. It is also possible to perform ptychography in pink beam configuration [25-27]. For data reconstruction the energy components are multiplexed and deconvoluted into different modes [26, 28, 29]. More recently it has been demonstrated that exploiting pink beam ptychography at I13 will allow for recording speeds of $10 \mathrm{kHz}$ [27]. Currently software, scanning capabilities and detectors are upgraded to explore the opportunity, and today we achieve an acquisition rate of $800 \mathrm{~Hz}$ in fly-scan mode, scanning $200 \mu^{2} \mathrm{~s}^{-1}$. Online and offline reconstructions are performed by PtyREX [2], the in-house developed software for ptychographic imaging taking into account the polychromaticity of the radiation spectrum and refining the sample positions in fly-scans. The most recent progress will reduce ptycho-tomography scanning times below an hour and larger amounts of samples will be scanned. We look forward to performing science on similar timescales as on the Imaging Branchline. For 
imaging with Bragg-CDI, exclusively the Si (111) monochromator was used (pink-beam scheme does not apply). Typical Bragg-CDI scans vary between 5 to 20 hours of total scanning time.

Figure 6 shows typical examples for research performed on batteries using ptycho-tomography and Braggptychography. The left figure shows a volume rendered from ptycho-tomographic data of one of the electrodes from a Samsung S7 battery. These batteries were reported for several failures resulting in device fires and were investigated by WMG as part of a battery forensic case study. The example on the right side shows a pre-study for imaging the phase changes of a single graphite particle of a Li-Ion anode during lithium intercalation in the crystal structure.
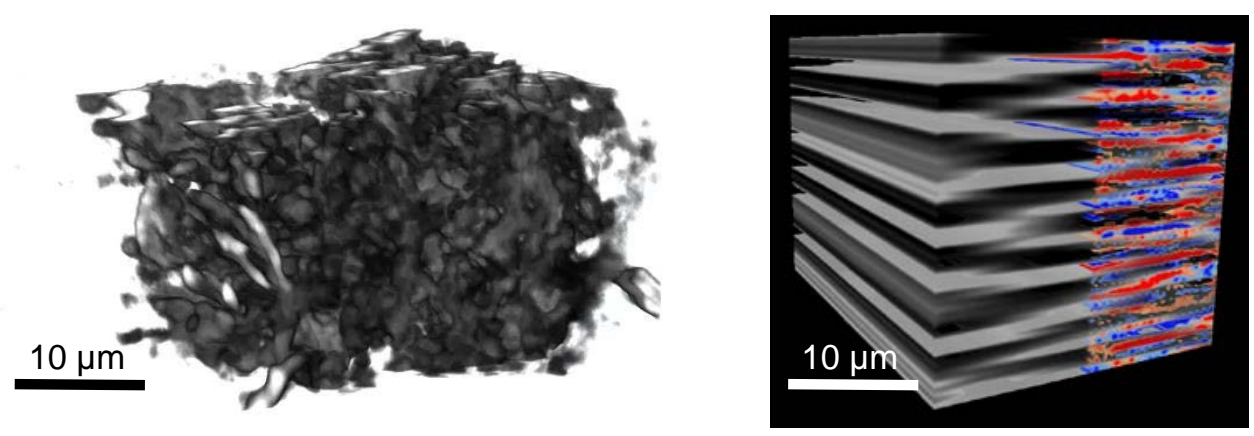

Figure 6: Examples of high-resolution tomography of battery materials. Left: Volume rendered tomo-ptychography data of a Samsung S7 battery electrode to investigate material failure (M. Loveridge, WMG, U. Warwick). Right: Reconstructed Bragg-ptychography data of a natural graphite crystal to investigate the electrode phase change by the dspacing expansion during lithium insertion (R. Ziesche, UCL).

\section{SUMMARY}

We provided examples of multi-scale research at I13L with micro- and nano-resolution. When working with energy bandwidth of $\Delta E / E=10^{-2}$ or pink beam, the recording time is significantly reduced which is important for in-situ and operando studies. While the feasibility of imaging with extended energy bandwidth has been tested for all methods at I13L, the instrumentation on the Coherence Branch is being developed to make full use of the potential capabilities. In future we expect to provide experimental capabilities for a complementary user program across both branchlines, covering resolutions from the nano- to the micron-range for samples between $100 \mu \mathrm{m}$ to $\mathrm{mm}$ size.

\section{ACKNOWLEDGEMENTS}

The authors especially thanks the teams of S. Schroeder (U. Leeds), M. Loveridge (Warwick Univ.), P. Shearing (UCL) and B. Connolly (Manchester Univ.) for sharing the results of their work. The Diamond-Manchester collaboration is acknowledged as well as the BP-ICAM/EPSRC Prosperity Partnership (EP/R00496X/1). The I13 team closely collaborates with C. David and Florian Döring (PSI) for X-ray optics. Kaz Wanelik, Andy Wilson and Ben Bradnick provide excellent support for beamline controls and data acquisition. Special thanks to our colleagues from the Diamond I24 beamline (R. Owen and D. Sherrell) for assistance with the scanning architecture at our beamline. The use of laboratory facilities available at the Research Complex at Harwell and with financial support from the Future Continuous Manufacturing and Advanced Crystallization (CMAC) Hub (EPSRC Grant EP/P006965/1) and for G. Das PhD studentship the University of Leeds are acknowledged.

\section{REFERENCES}

[1] Atwood, R. C., Bodey, A. J., Price, S. W. T., Basham, M., and Drakopoulos, M., “A high-throughput system for high-quality tomographic reconstruction of large datasets at Diamond Light Source," Philos. Trans. R. Soc. Ser. A, 373(2043), 20140398 (2015).

[2] Batey, D. J., "PtyREX: A user-friendly software package for reconstructing ptyhcographic data recorded with electrons, X-rays and visble light,” (in preparation).

[3] Bodey, A. J., and Rau, C., "Launch of the I13-2 data beamline at the Diamond Light Source synchrotron," Journal of Physics Conference Series, 849, 012038 (2017).

[4] Rau, C., Wagner, U., Pesic, Z., and De Fanis, A., “Coherent imaging at the Diamond beamline I13,” Physica Status Solidi a-Applications and Materials Science, 208(11), 2522-2525 (2011). 
[5] Rau, C., Wagner, U. H., Vila-Comamala, J., Bodey, A., Parson, A., Garcia-Fernandez, M., De Fanis, A., and Pesic, Z., "Imaging in Real and Reciprocal Space at the Diamond Beamline I13," AIP Conference Proceedings, 1696, 020047 (2016).

[6] Rau, C., Weitkamp, T., Snigirev, A., Schroer, C., Benner, B., Tummler, J., Gunzler, T., Kuhlmann, M., Lengeler, B., Krill, C., Doebrich, K., Michels, D., and Michels, A., "Tomography with high resolution," Proceedings of the SPIE, 4503, 14-22 (2002).

[7] Rack, A., Morawe, C., Mancini, L., Dreossi, D., Parkinson, D. Y., Mac Dowell, A. A., Siewert, F., Rack, T., Holz, T., Kramer, M., and Dietsch, R., "Reflection on multilayer mirrors: beam profile and coherence properties," Proceedings of SPIE, 9207, 92070V (2014).

[8] Rack, A., Vivo, A., Helfen, L., and Morawe, C., "Beam profile and coherence properties of synchrotron beams after reflection on modified multilayer mirrors," AIP Conference Proceedings, 1741, 040026 (2016).

[9] Andrew, M., Menke, H., Blunt, M. J., and Bijeljic, B., "The Imaging of Dynamic Multiphase Fluid Flow Using Synchrotron-Based X-ray Microtomography at Reservoir Conditions,” Transport in Porous Media, 110(1), 1-24 (2015).

[10] Singh, K., Menke, H., Andrew, M., Lin, Q. Y., Rau, C., Blunt, M. J., and Bijeljic, B., "Dynamics of snap-off and pore-filling events during two-phase fluid flow in permeable media," Scientific Reports, 7, 5192 (2017).

[11] Menke, H. P., Andrew, M. G., Vila-Comamala, J., Rau, C., Blunt, M. J., and Bijeljic, B., "Dynamic Pore-scale Reservoir-condition Imaging of Reaction in Carbonates Using Synchrotron Fast Tomography,” J. Vis. Exp.(120), e53763 (2017).

[12] Menke, H. P., Reynolds, C. A., Andrew, M. G., Nunes, J. P. P., Bijeljic, B., and Blunt, M. J., “4D multi-scale imaging of reactive flow in carbonates: Assessing the impact of heterogeneity on dissolution regimes using streamlines at multiple length scales," Chemical Geology, 481, 27-37 (2018).

[13] Reynolds, C. A., Menke, H., Andrew, M., Blunt, M. J., and Krevor, S., "Dynamic fluid connectivity during steady-state multiphase flow in a sandstone," Proceedings of the National Academy of Sciences of the United States of America, 114(31), 8187-8192 (2017).

[14] Momose, A., Kawamoto, S., Koyama, I., Hamaishi, Y., Takai, K., and Suzuki, Y., "Demonstration of X-Ray Talbot interferometry,” Japanese Journal of Applied Physics Part 2-Letters \& Express Letters, 42(7B), L866L868 (2003).

[15] Weitkamp, T., Diaz, A., David, C., Pfeiffer, F., Stampanoni, M., Cloetens, P., and Ziegler, E., "X-ray phase imaging with a grating interferometer,” Optics Express, 13(16), 6296-6304 (2005).

[16] Zdora, M. C., Vila-Comamala, J., Schulz, G., Khimchenko, A., Hipp, A., Cook, A. C., Dilg, D., David, C., Grunzweig, C., Rau, C., Thibault, P., and Zanette, I., "X-ray phase microtomography with a single grating for high-throughput investigations of biological tissue,” Biomedical Optics Express, 8(2), 1257-1270 (2017).

[17] Marathe, S., Zdora, M. C., Zanette, I., Cipiccia, S., and Rau, C., "Comparison of Data Processing Techniques for Single Grating X-ray Talbot Interferometer Data," Proceedings of SPIE, 10391, 103910S (2017).

[18] Bikis, C., Rodgers, G., Deyhle, H., Thalmann, P., Hipp, A., Beckmann, F., Weitkamp, T., Theocharis, S., Rau, C., Schulz, G., and Muller, B., "Sensitivity comparison of absorption and grating-based phase tomography of paraffin-embedded human brain tissue,” Applied Physics Letters, 114(8), 083702 (2019).

[19] Rodgers, G., Schulz, G., Deyhle, H., Marathe, S., Bikis, C., Weitkamp, T., and Muller, B., "A quantitative correction for phase wrapping artifacts in hard X-ray grating interferometry,” Applied Physics Letters, 113(9), 093702 (2018).

[20] Marathe, S., "Development of synchrotron pink beam X-ray grating interferometer for fast imaging and tomography applications," 11113, 1111319.

[21] Stampanoni, M., Mokso, R., Marone, F., Vila-Comamala, J., Gorelick, S., Trtik, P., Jefimovs, K., and David, C., "Phase-contrast tomography at the nanoscale using hard x rays," Physical Review B, 81(14), 140105(R) (2010).

[22] Vila-Comamala, J., Bosgra, J., Eastwood, D. S., Wagner, U., Bodey, A. J., Garcia-Fernandez, M., David, C., and Rau, C., "Transmission X-ray Microscopy at Diamond-Manchester I13 Imaging Branchline," AIP Conference Proceedings, 1696, 020036 (2016).

[23] Storm, M., Cipiccia, S., Marathe, S., Kuppili, V. S. C., Doring, F., David, C., and Rau, C., "The Diamond I13-2 Transmission X-ray Microscope: Current Status and Future Developments,” Microscopy and Microanalysis, 24(S2), 216-217 (2018).

[24] Jefimovs, K., Vila-Comamala, J., Stampanoni, M., Kaulich, B., and David, C., "Beam-shaping condenser lenses for full-field transmission X-ray microscopy,” Journal of Synchrotron Radiation, 15, 106-108 (2008). 
[25] Edo, T. B., Batey, D. J., Maiden, A. M., Rau, C., Wagner, U., Pesic, Z. D., Waigh, T. A., and Rodenburg, J. M., "Sampling in x-ray ptychography," Physical Review A, 87(5), 053850 (2013).

[26] Batey, D. J., Edo, T. B., Rau, C., Wagner, U., Pesic, Z. D., Waigh, T. A., and Rodenburg, J. M., "Reciprocalspace up-sampling from real-space over sampling in x-ray ptychography,” Physical Review A, 89(4), 043812 (2014).

[27] Cipiccia, S., Batey, D., Shi, X., Williams, S., Wanelik, K., Wilson, A., Martin, P., Scott, T., and Rau, C., "Multi-scale multi-dimensional imaging at I13-coherence branchline in Diamond Light Source," AIP Conference Proceedings, 2054, 050005 (2019).

[28] Batey, D. J., Claus, D., and Rodenburg, J. M., “Information multiplexing in ptychography,” Ultramicroscopy, 138, 13-21 (2014).

[29] Thibault, P., and Menzel, A., "Reconstructing state mixtures from diffraction measurements," Nature, 494(7435), 68-71 (2013). 